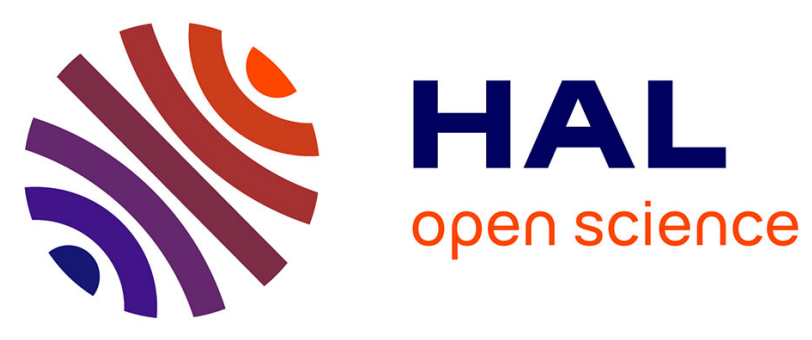

\title{
Improving Silicon Nitride Ring Resonator Performances On 300 mm Industrial Environment For Point of Care Applications
}

\author{
Michele Calvo, Guillaume Beaudin, Laurence Mercier-Coderre, Pauline \\ Girault, Pedro Rojo Romeo, Romain Stricher, Serge Ecoffey, Dominique \\ Drouin, Frederic Boeuf, Michael Canva, et al.
}

\section{To cite this version:}

Michele Calvo, Guillaume Beaudin, Laurence Mercier-Coderre, Pauline Girault, Pedro Rojo Romeo, et al.. Improving Silicon Nitride Ring Resonator Performances On $300 \mathrm{~mm}$ Industrial Environment For Point of Care Applications. Biophotonics in Point-of-Care, Apr 2020, Online Only, France. pp.5, 10.1117/12.2555339 . hal-03517861

\section{HAL Id: hal-03517861 \\ https://hal.science/hal-03517861}

Submitted on 8 Jan 2022

HAL is a multi-disciplinary open access archive for the deposit and dissemination of scientific research documents, whether they are published or not. The documents may come from teaching and research institutions in France or abroad, or from public or private research centers.
L'archive ouverte pluridisciplinaire HAL, est destinée au dépôt et à la diffusion de documents scientifiques de niveau recherche, publiés ou non, émanant des établissements d'enseignement et de recherche français ou étrangers, des laboratoires publics ou privés. 


\title{
Improving Silicon Nitride Ring Resonator Performances On 300 mm Industrial Environment For Point of Care Applications
}

\author{
Michele Calvo*a,b,c,d , Guillaume Beaudin ${ }^{\mathrm{b}, \mathrm{c}}$, Laurence Mercier-Coderre ${ }^{\mathrm{b}, \mathrm{c}}$, Pauline Girault ${ }^{\mathrm{b}, \mathrm{c}}$, Pedro \\ Rojo Romeo $^{\mathrm{b}, \mathrm{c}, \mathrm{d}}$, Romain Stricher ${ }^{\mathrm{b}, \mathrm{c}}$, Serge Ecoffey ${ }^{\mathrm{b}, \mathrm{c}}$, Dominique Drouin ${ }^{\mathrm{b}, \mathrm{c}}$, Frederic Bœuf ${ }^{\mathrm{a}}$, Michael \\ Canva $^{\mathrm{b}, \mathrm{c}}$, Stephane Monfray ${ }^{\mathrm{a}}$, Regis Orobtchouk ${ }^{\mathrm{d}}$, Paul G.Charette $\mathrm{e}^{\mathrm{b}, \mathrm{c}}$ \\ ${ }^{a}$ STMicroelectronics, 850 Rue Jean Monnet, Crolles, 38920, France \\ bLaboratoire Nanotechnologies Nanosystèmes (LN2)-CNRS UMI-3463, Université de Sherbrooke, \\ Sherbrooke, Québec, J1K 0A5, Canada \\ 'Institut interdisciplinaire d'innovation technologique (3IT), Université de Sherbrooke, Sherbrooke, \\ Québec, J1K 0A5, Canada \\ 'Institut des Nanotechnologies de Lyon (INL), CNRS UMR 5270, Université de Lyon, INSA-Lyon, \\ Bât "Blaise Pascal", 7 avenue Jean Capelle, Villeurbanne, F-69621, France
}

\begin{abstract}
Point-of-care tests (POCT) are important for detecting illnesses and monitoring patients without the need of a medical laboratory. To be useful, POCT must be sensitive, specific, integrated, and affordable. Since the early 2000s, integrated photonics have offered a possible solution for this problem. In particular, silicon micro-ring resonators represent a compact and sensitive choice known in the industry. This paper details the design, fabrication, and characterization of two methods for improving the performance of ring resonators by engineering their cross section. More precisely, improving devices made out of silicon nitride in an industrial environment to work in the infrared (around $1.31 \mu \mathrm{m})$.
\end{abstract}

Keywords: Biosensors, lab-on-chip, biophotonics, plasmonics, higher order modes.

\section{INTRODUCTION}

To be effective, POCT devices have to be sensitive, compactness, low cost and compatible with functionalization process. This paper shows two alternative approaches to integrated nano-photonic sensing for point of care testing[1], [2]. The proposed structures, demonstrate not only a higher sensitivity, but consider selectivity and manufacturing issues, fundamental for POCT development. Concerning the low cost aspect, the devices were made in an industrial environment Datacom Advanced PHotonic Nanoscale Environment (DAPHNE) which allowed a high throughput and repeatability. The two approaches, Hybrid Plasmonic WaveGuides and the higher order modes, are listed below and each section describes the differences and the common points.

\subsection{Hybrid Plasmonic waveguides}

Hybrid plasmonic waveguides, or HPWG, are partially made of a plasmonic waveguide and partially made of a dielectric waveguide. Picture 1.a shows the cross section of a hybrid plasmonic waveguide. The vertical walls of the waveguide support a plasmonic mode only with a polarization perpendicular to its surface. The dielectric waveguide, although it can support polarization in both directions, it should be polarized in the same direction as the plasmonic waveguide to maximize the coupling between the two. The polarization direction of the field of the separate modes is shown in picture 1. The geometry of the HPWG can be engineered to maximize the power deconfinement from the dielectric waveguide into the target liquid. The HPWG improves designed in this paper improves the sensitivity of the sensor with respect to a dielectric sensor. Furthermore, the sensor is made of a dielectric and a metallic part, which makes the functionalization easier to achieve for analyte detection applications.

The materials making up the waveguide are $\mathrm{SiN}$ and $\mathrm{SiO} 2$ because they are compatible with the industrial fabrication. The plasmonic waveguide was chosen to be Au because of its plasmonic aspect, its compatibility with biological application and its availability both in the university and industrial cleanroom. 


\subsection{Higher order modes}

Higher order mode sensors approach consists in selectively excite the first order mode of the ring resonator's waveguide. An example of cross section is shown in figure 1.b. The higher order mode is designed to be close to its cutoff. Thanks to its greater exposure to the sensing liquid than the fundamental mode, first order modes are more sensitive. Furthermore, the first order waveguide constituting the device is multimode, thus wider than a monomode one $(1 \mu \mathrm{m}$ and $0.5 \mu \mathrm{m}$ respectively). This greater width increases the exposed surface and reduces the constraints on fabrication especially concerning the lithography resolution. Thus the waveguides are easier to make and have lower impact on fabrication errors.

In the proof of concept in this paper, the first order mode is selectively excited by using a monomode bus waveguide with a fundamental mode having the same effective refractive index. The monomode and the multimode waveguide couple generating even and odd modes as shown in the simulation section.

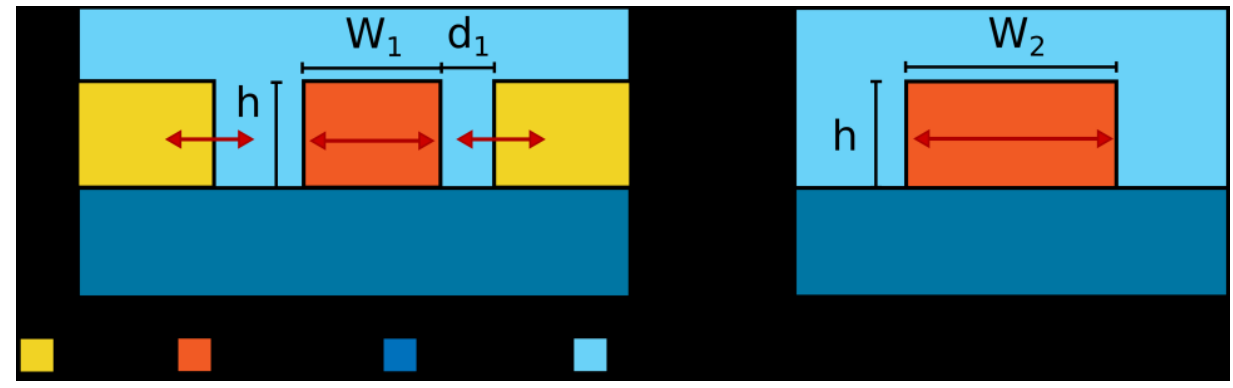

Figure 1 Cross section of a HPWG and a second order mode waveguide made of silicon nitride with the industrial DAPHNE platform developed at STMicroelectronics

\section{SIMULATION}

Photonic devices studied in this paper were simulated by a custom design mode solver verified with FIMMWAVE simulation software. Once the mode of the waveguide and its effective refractive index was found, the whole ring resonator was simulated thanks to the analytical expression of the transmission of the ring. Next two sections present the simulation results for the proposed geometry: the hybrid plasmonic waveguide or HPWG and the higher order mode ring resonator.

\subsection{Hybrid plasmonic waveguides}

Hybrid plasmonic waveguides cross section geometry and simulation is shown in figure 2.a and 2.b. It can be noticed that simulations confirm that the coupling between the plasmonic walls and the dielectric waveguide takes place. In fact, the field distribution shown in figure 2.b shows a high energy in the gap between the Au and the SiN, in the sensing liquid. The filling factor in the sensing liquid, i.e. the percentage of the field in the sensing liquid, is of about $60 \%$ for the geometry described in the picture.

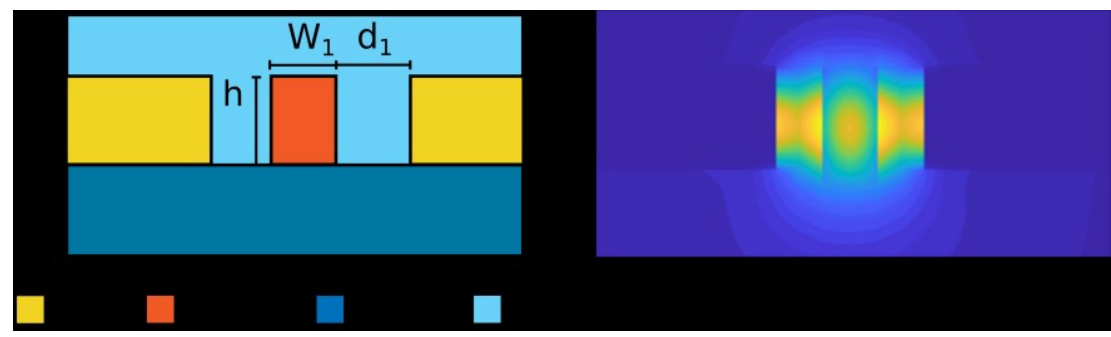

Figure 2 Cross section of the HPWG waveguide and its simulation of the its propagation mode for $h=600 \mathrm{~nm}$, $W_{1}=300 \mathrm{~nm}$ and $d_{1}=300 \mathrm{~nm}$. 
This high percentage increases the shift of effective refractive index of the mode and by consequences the resonance peak shift of the ring resonator. Simulations give a sensibility of $550 \mathrm{~nm} / \mathrm{RIU}$ (RIU = refractive index unit, it is the unit of the refractive index which in the international system is dimensionless). However, HPWG also have large losses with respect to a dielectric waveguide due to the high absorption coefficient of the metal. Furthermore, in a ring resonator the bend of the waveguide can cause deconfinement effects on the propagation of the mode. For this reason the shape of the ring resonator is a racetrack where only the straight parts where HPWG. The percentage of the ring to be metalized is from $10 \%$ to $30 \%$ based on the geometry. The simulated sensitivity considering the dielectric bends is around $270 \mathrm{~nm} / \mathrm{RIU}$. As expected it is lower than the sensitivity of a full hybrid plasmonic ring, because the bends are dielectric waveguides, thus less sensitive.

\subsection{Higher order modes}

The modes of monomode and multimode waveguides are shown in figure 3.a and 3.b. The higher order mode has two power peaks in the waveguide and its power in the sensing liquid is more important that the one of a fundamental mode. This shows that the first order mode is more sensitive with respect to the fundamental.

However, simply increasing the width of a waveguide brings up another problem: the first order mode has to be selectively excited so that no power leaks to other modes, especially for the fundamental. The price to pay if the fundamental mode is a decrease in sensitivity, increase incertitude and may cause double resonances in the ring complicating the detection. The system that is used in this paper to avoid excitation of the first order is through asymmetrical directional couplers: one of the waveguide is monomode and the other is multimode. They are designed to have the same refractive index of the modes. In this case, coupling is very low from the two fundamental modes of the waveguides while the preferential one is the one between the fundamental mode of the monomode waveguide and the first order mode of the multimode. Figure 3.c shows how the electric field of the two waveguides is influenced by the presence of the other one. Critical coupling conditions are achieved by choosing the distance between the waveguides.Like with HPWG, a racetrack ring resonators is used to change the phase shift caused by the analyte into intensity. The sensitivity found in the simulation is of $250 \mathrm{~nm} / \mathrm{RIU}$.

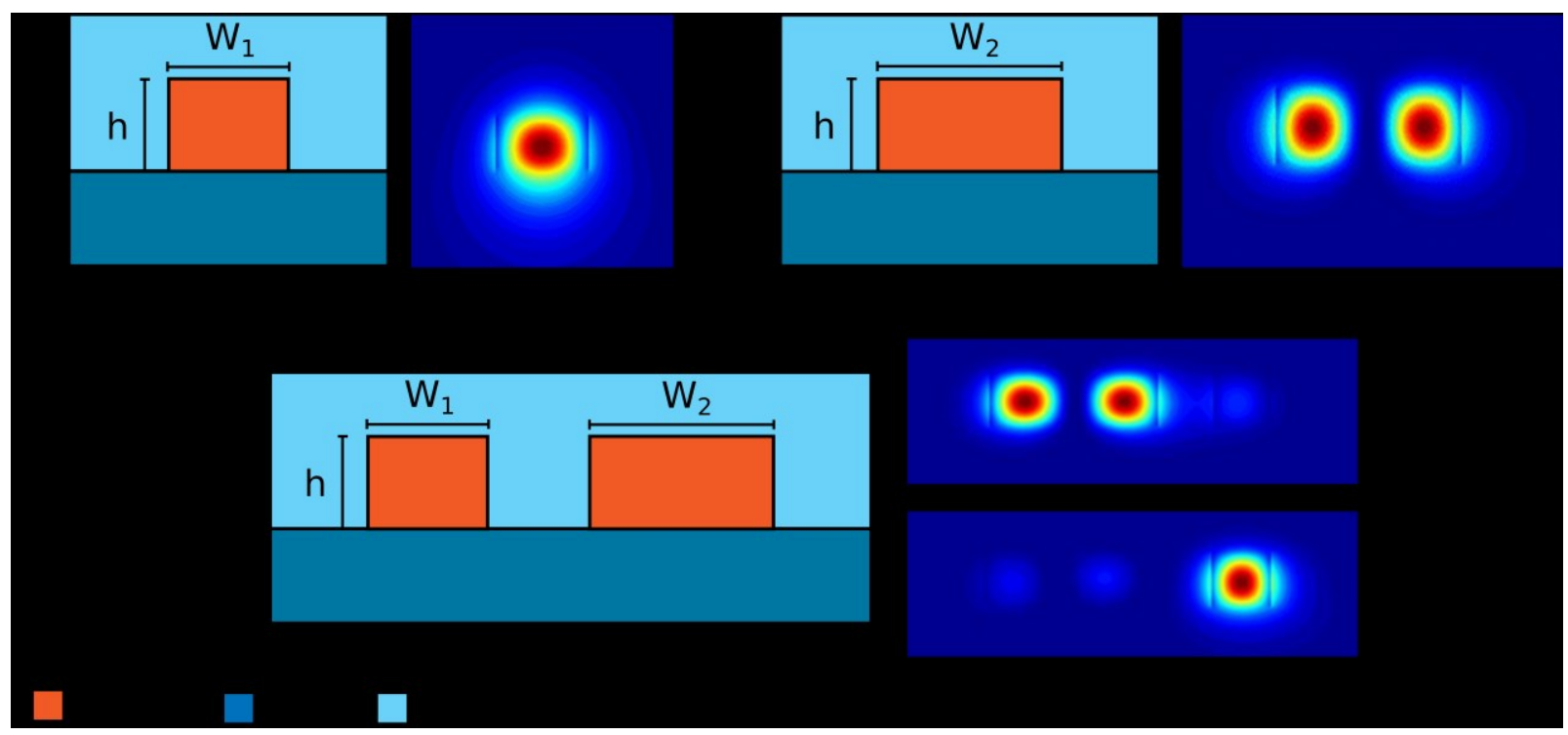

Figure 3.a monomode waveguide mode. 3.b two-modes waveguide where the first order mode is simulated. Its effective index is 1.45, close to the cutoff. 3.c shows the two modes close to each other and the coupling between the two modes having similar index of refraction. 


\section{FABRICATION AND SETUP}

The fabrication of the $\mathrm{SiN}$ waveguides and the $\mathrm{SiO} 2$ is performed in an industrial environment called Datacom Advanced PHotonic Nanoscale Environment or DAPHNE. Working in an industrial environment is important because one of the key points of POCT is that the production per device should be cheap. It is then paramount to already work with in the condition of a $300 \mathrm{~mm}$ SOI industrial platform[3].

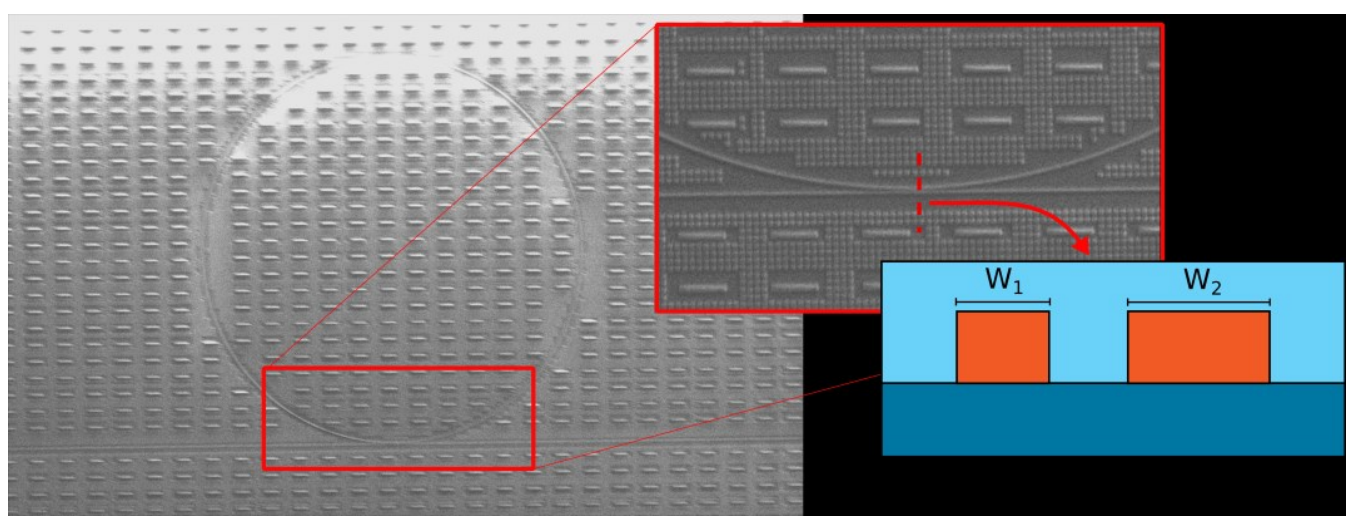

Figure 5 Scanning electron microscope image of the higher order ring resonator. A zoom on the coupling section is shown as well as the schematics showing the cross section.

The fabrication of the HPWG sensors needs the Au to be added. Although available in the industrial environment, the metal was added in the university cleanroom with the lift-off process, not compatible with the industrial platform, depicted in figure 4.a. Once the metal is tested, another method not presented in this paper is developed to have a compatible manufacturing with the industry. The final result of the ring resonator is shown in the scanning electron microscopy image figure 4.b.

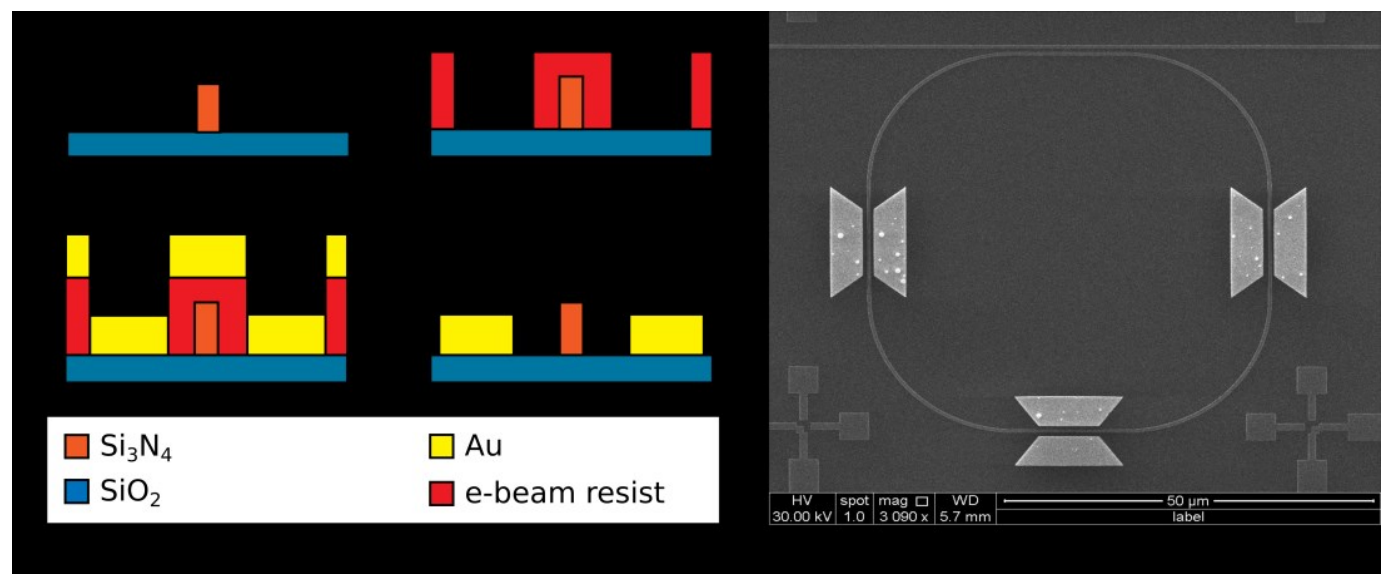

Figure 4.a image of the process to build a HPWG, lift-off. 4.b scanning electron microscopy image of the manufactured ring resonator

Once the rings were manufactured at STMicroelectronics, a polydimethylsiloxane (PDMS) ring was glued to the device surface to contain the fluids used during measurements. Figure 6.a show the sample with the empty PDMS reservoir.

The schematics of the setup is shown in picture 6.b. The input laser (Agilent 81682A tunable laser, 1.46 to $1.58 \mu \mathrm{m}$ wavelength range, $0.1 \mathrm{pm}$ resolution) is followed by a polarization control device which selects TE light in this case. Using a lensed fiber, light is then injected into the bus waveguide. A photodetector (Agilent 81618A with 81624 head, 0.8 to $1.7 \mu \mathrm{m}$ wavelength range, -90 to $+10 \mathrm{dBm}$ power range) measures the optical power from a lensed fiber coupled to the output bus waveguide. Figure 7.c shows the devices during the experiment. The inset shows a zoom on the sample in 
the adapted sample holder. Different concentrations of glucose monohydrate in $\mathrm{H}_{2} \mathrm{O}$ were used to test the bulk response of the device: from $2 \mathrm{~g} / 100 \mathrm{ml}$ to $6 \mathrm{~g} / 100 \mathrm{ml}$, in refractive index from $2 \times 10^{-3}$ to $7 \times 10^{-3} \mathrm{RIU}$ (refractive index unit).

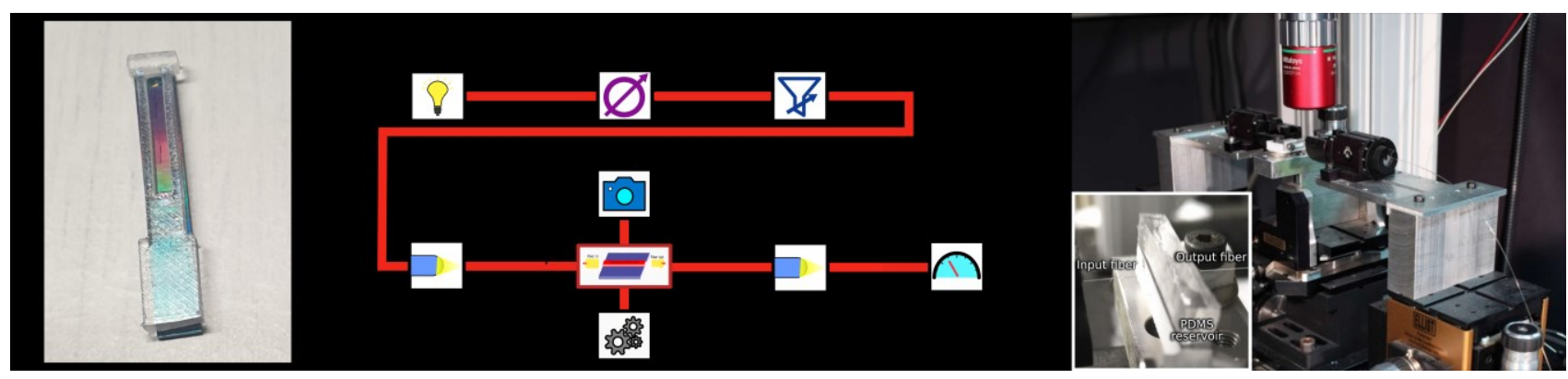

Figure 5 a schematics of the setup. 5.b setup with the chip and the PDMS reservoir. 5.c setup while charging the reservoir with the syringe.

\section{EXPERIMENTAL RESULTS}

This section presents the experimental results. These results are compared to a conventional ring resonator, built with the same platform with a rib waveguide. This reference ring has a sensitivity of $120 \mathrm{~nm} / \mathrm{RIU}$.

\subsection{Hybrid plasmonic waveguides}

The transmission of the HPWG resonator is shown in figure 7.a. The quality factor is low (around 500) because of the presence of the metal. Furthermore, the algorithm to calculate the peak position is a fit with a Lorentzian curve to find the minimum and the associated uncertainty [4]. As expected, the sensitivity of the HPWG device is around $275 \mathrm{~nm} / \mathrm{RIU}$. This value is calculated as the slope of the calibration curve. Error bars are found by using the combined standard uncertainty that links the temperature uncertainty, the refractive index uncertainty and fabrication uncertainty. The results of HPWG calibration curve are shown in picture 7.b. The experimental sensitivity value is different from the simulated one because the alignment of the metal was not perfectly symmetric.
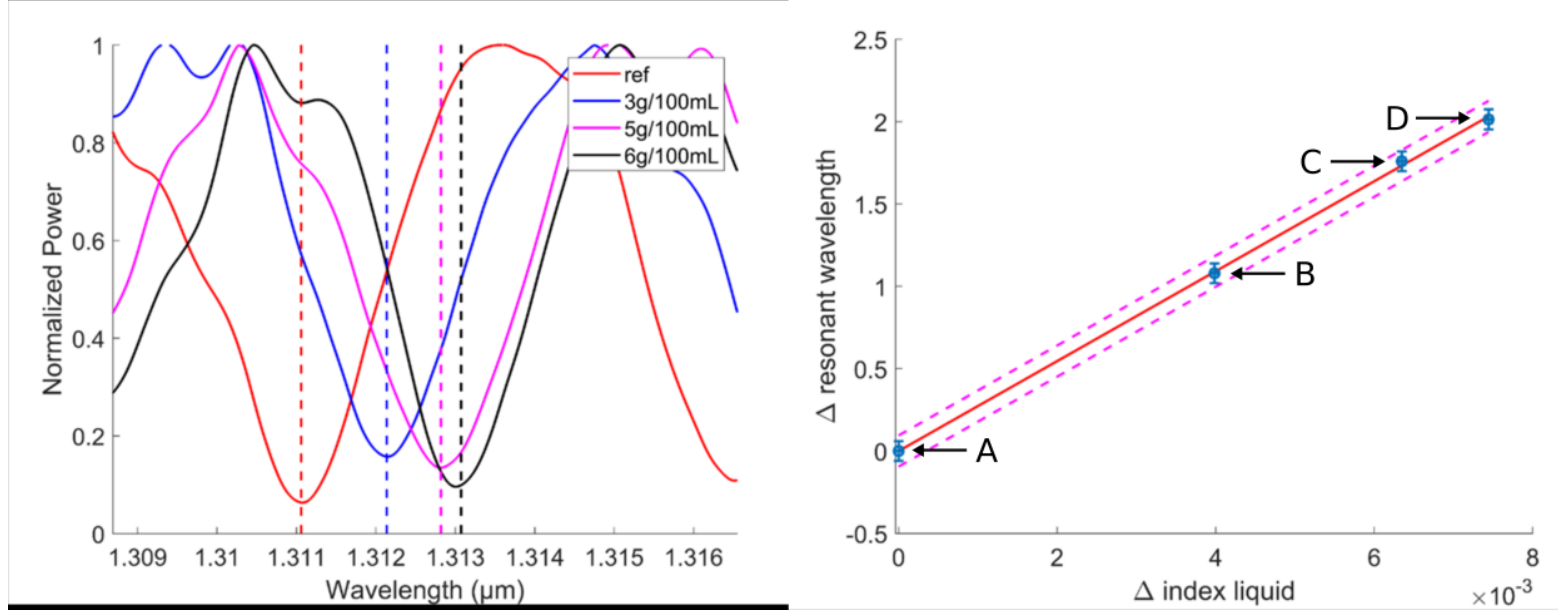

Figure 7.a Transmission spectrum of the HPWG device for different glucose monohydrate dilutions in H2O. 7.b Calibration curve to calculate the sensitivity as the slope of the fit line. A represent the reference wavelenght, $B$ the $3 \mathrm{~g} / 100 \mathrm{~mL}, C$ the $5 \mathrm{~g} / 100 \mathrm{~mL}$ and $D$ the $6 \mathrm{~g} / 100 \mathrm{~mL}$. Sensitivity is of $275 \mathrm{~nm} / \mathrm{RIU}$. 


\subsection{Higher order modes}

The transmission curves of the higher order ring resonator are shown in figure 8.a. The quality factor is higher than in the case of HPWG (around 5000) because there is no metal. The sensitivity of the sensor was found to be around $212 \mathrm{~nm} / \mathrm{RIU}$. This value is slightly lower than the simulation because of fabrication tolerances.
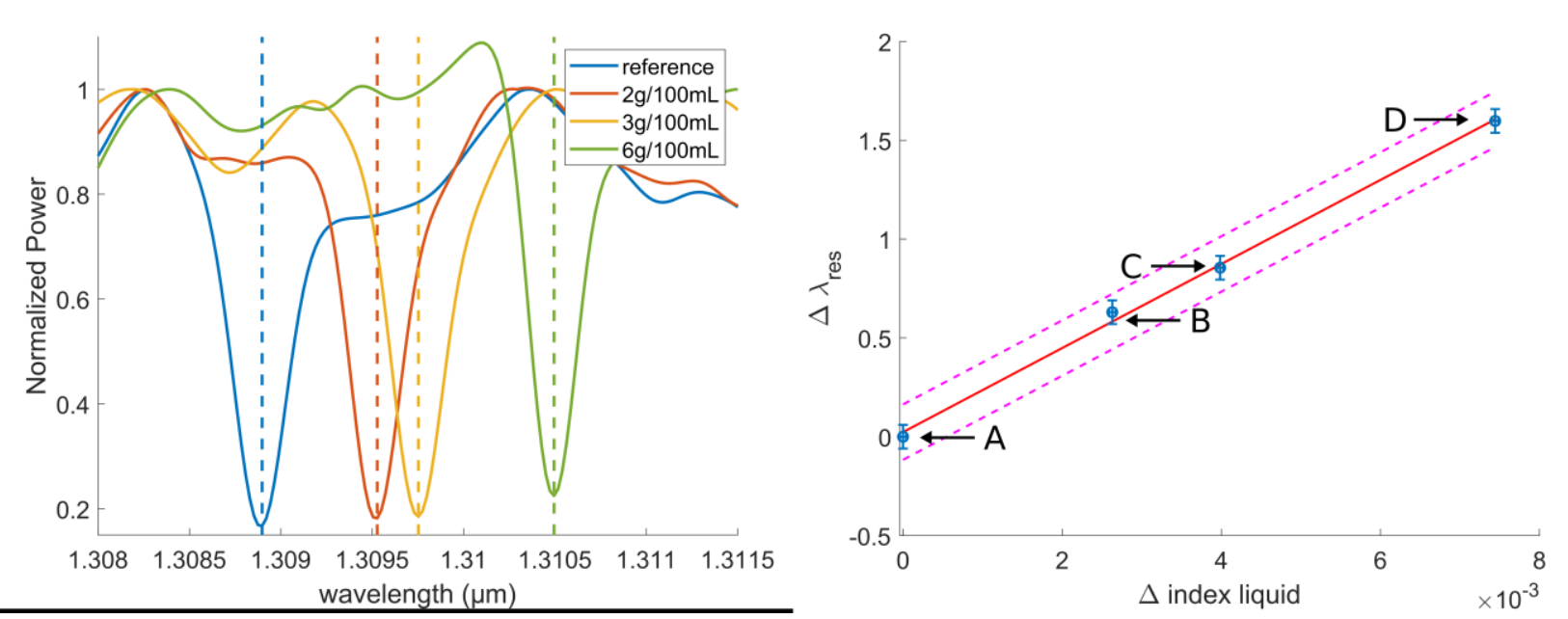

Figure 8.a transmissions for the higher order mode device for different glucose monohydrate dilutions in $\mathrm{H} 2 \mathrm{O}$. 8.b Calibration curve to calculate the sensitivity as the slope of the fit line. A represent the reference wavelenght, $B$ the $2 \mathrm{~g} / 100 \mathrm{~mL}, C$ the $3 \mathrm{~g} / 100 \mathrm{~mL}$ and $D$ the $6 \mathrm{~g} / 100 \mathrm{~mL}$. Sensitivity is of $212 \mathrm{~nm} / \mathrm{RIU}$.

\section{CONCLUSION}

Two approaches to improve the sensitivity of a silicon nitride waveguide are shown. Both approaches are compatible with the cleanroom environment, which is fundamental for a lab-on-chip device. Moreover both devices have been tested with different refractive index liquids and they show a higher sensitivity compared to a rib waveguide (120nm/RIU). The perspective of the HPWG resonator is to improve the sensitivity by increasing the hybrid plasmonic part in the ring.

\section{REFERENCES}

[1] A. E. Cetin et al., "Hand-Held plasmonic biosensor for high-throughput sensing for point-of-care applications," in Lasers and Electro-Optics (CLEO), 2014 Conference on, 2014, pp. 1-2.

[2] L. Chrostowski et al., "Silicon photonic resonator sensors and devices," presented at the Laser Resonators, Microresonators, and Beam Control XIV, 2012, vol. 8236, p. 823620, doi: 10.1117/12.916860.

[3] M. Calvo et al., "Ring Resonator Designed for Biosensing Applications Manufactured on $300 \mathrm{~mm}$ SOI in an Industrial Environment," Solid States Devices and Materials 2018, vol. Solid States Devices and Materials 2018, p. 2 .

[4] T. Claes, W. Bogaerts, and P. Bienstman, "Experimental characterization of a silicon photonic biosensor consisting of two cascaded ring resonators based on the Vernier-effect and introduction of a curve fitting method for an improved detection limit," Opt. Express, OE, vol. 18, no. 22, pp. 22747-22761, Oct. 2010, doi:

10.1364/OE.18.022747. 